\title{
The Ethnopharmacological Literature: An Analysis of the Scientific Landscape in the Cerrado in Central-Western Brazil
}

\author{
Sidney Mariano dos Santos ${ }^{1}$, Kamilla Felipe do Nascimento ${ }^{1}$, Zefa Valdevina Pereira ${ }^{1}$, \\ Josimo Diego Bazanella Line ${ }^{1}$, Pedro Cruz de Oliveira Junior ${ }^{1}$, Janaine Alberto Marangoni ${ }^{1}$, \\ Maria do Carmo Vieira ${ }^{1}$, Rosilda Mara Mussury Franco Silva ${ }^{1} \&$ Anelise Samara Nazari Formagio ${ }^{1}$ \\ ${ }^{1}$ Federal University of Grande Dourados, MS, Brazil \\ Correspondence: Anelise Samara Nazari Formagio, Federal University of Grande Dourados, MS, Brazil. Tel: \\ 67-98128-3141. E-mail: aneliseformagio@gmail.com
}

Received: July 21, 2020

doi:10.5539/jas.v12n11p307
Accepted: August 31, $2020 \quad$ Online Published: October 15, 2020

URL: https://doi.org/10.5539/jas.v12n11p307

\begin{abstract}
Research on pharmacology and phytochemistry originating from medicinal plants has resulted in various publications highlighting the Cerrado in central-western Brazil, which has a remarkable diversity of plant species. The reserve area selected was the Cerrado stricto sensu settlement "17 April", Mato Grosso do Sul (MS), Brazil. However, no ethnopharmacological review focusing on the plants present in the reserve area exists, even though the consumption of medicinal plants is a widespread practice. The aims of this study were to 1) survey and document the medicinal plants present in the reserve area; 2) provide an overview of recent ethnopharmacological, phytochemical and pharmacological studies of these species; and 3) provide insight for future studies. A literature search was conducted, and relevant information was collected from authentic resources using databases such as Science Direct, PubMed, Google Scholar, Web of Science and Scopus, as well as peer reviewed articles, books and theses. Eighty-nine species belonging to 39 different families were found; the most abundant were Fabaceae $(n=13)$, Myrtaceae $(n=7)$, Rubiaceae $(n=7)$ and Bignoniaceae $(n=5)$. In terms of it empirical use, the most utilized parts were leaves $(41 \%)$, bark $(22 \%)$ and roots $(15 \%)$. The most widespread traditional use, according to the literature review of the following plants involves the treatment of gastro-intestinal system diseases (41 spp). Chemical studies reported a high presence of terpene, phenol, and alkaloid classes. Only three are listed in the RENISUS: Casearia sylvestris, Copaifera langsdorffii and Stryphnodendron adstringens. This study demonstrated a large number of medicinal plants in an area of the Cerrado in the state of Mato Grosso do Sul, Brazil. Noting the importance of biodiversity for the development of new pharmacological approaches, many studies prove the empirical use of medicinal plants.
\end{abstract}

Keywords: Cerrado, Mato Grosso do Sul, medicinal plants, review

\section{Introduction}

Brazil is characterized by some of the greatest biodiversity on the planet due to the presence of different biomes, such as the Amazon (tropical forest), Caatinga (thorn forest), Pantanal (flooded pasture), Pampas (subtropical pastures or pastures), Atlantic Forest (deciduous forest) and Cerrado (savanna) (Guerra et al., 2020). The Cerrado is the second largest biome in South America, occupying an area of approximately $22 \%$ of the Brazilian territory, and had the second greatest biodiversity richness (Sano et al., 2019). The state of Mato Grosso do Sul is located in midwestern Brazil, and most of its territory is occupied by the Cerrado (Amaral et al., 2017). Nevertheless, $46 \%$ of the original Cerrado area has been converted to pasture and cropland and is continually threatened by the indiscriminate use of fire (Durigan \& Ratter, 2015; Strassburg et al., 2014).

The study of ethnopharmacology is closely related to sustainable development, as it is an effective way to develop medicines from the perspective of traditional plant use. In this sense, the importance of studies in this area is clear, as they contribute to the improvement of the traditional application of natural products, and they emphasize the importance of biodiversity for the sustainability of local populations (Di Stasi et al., 2002). In this context, the Brazilian government encourages the use of herbal remedies and medicinal plants as a strategy to improve the use of Brazil's biodiversity and public access to herbal medicines.

In this study, we conducted a 1) literature survey and documented the medicinal species present in the reserve area of the settlement "April 17", MS, Brazil, in the Cerrado biome; 2) Exhaustive research has evaluated the 
current status of scientific knowledge related to the popular use and phytochemical and pharmacological properties of these species; 3) We also discuss the species found in the study area that are noted in research on herbal medicines. The population residing in this area is characterized by agriculture and their means of subsistence, with different natural resources used for different purposes. Considering the abundant vegetation and the scarcity of financial resources, the local population often uses natural resources to treat diseases without any knowledge of scientific evidence. After the identification of all the species found in this area, a survey of scientific studies involving these species is absolutely relevant and necessary, aiming to contribute to the safety and well-being of those who commonly use these plants, both in the target region of this study and in others.

\section{Material and Methods}

\subsection{Study Sites}

In the framework of this study, the selected area, Cerrado stricto sensu $\left(21^{\circ} 46^{\prime} 54.6^{\prime \prime} \mathrm{S}\right.$ and $\left.53^{\circ} 13^{\prime} 23.6^{\prime \prime} \mathrm{W}\right)$, comprised the reserve area of the settlement "17 April", located in Casa Verde district, Nova Andradina city, MS (Figure 1).

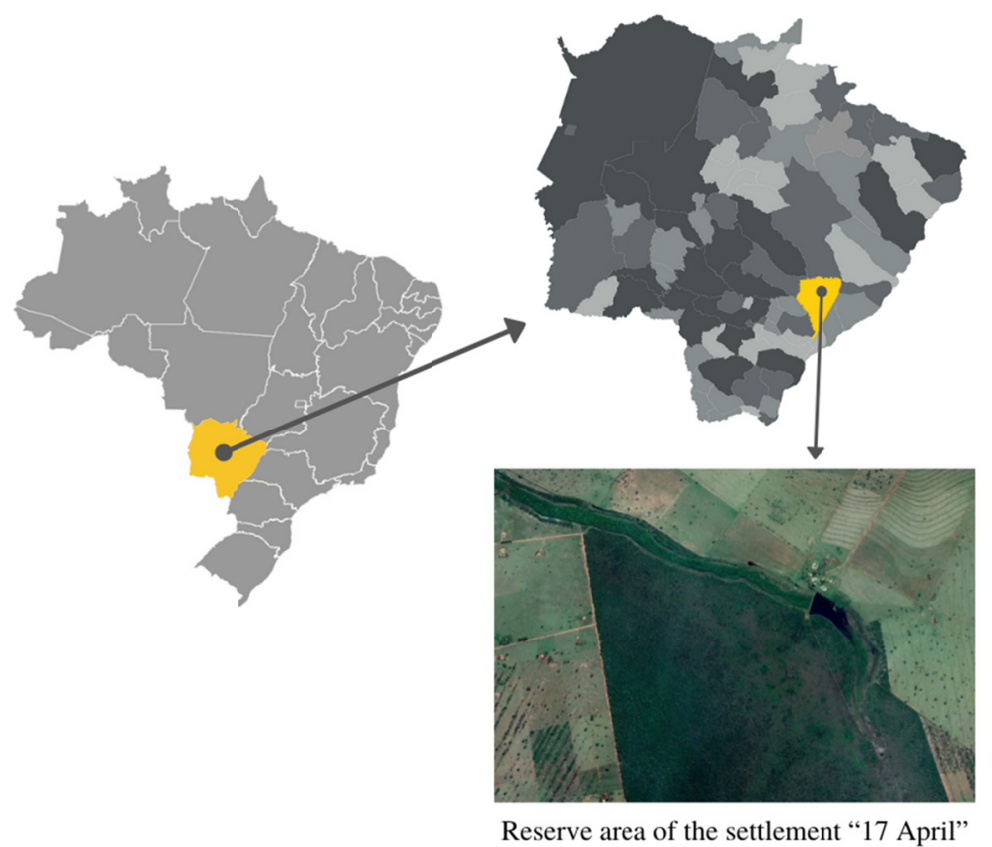

Figure 1. Geographical location of the study area (reserve area of the settlement "17 April")

\subsection{Collection and Identification of Species}

The collections of the species present in this area were carried out between 09/2017 and 01/2018. Botanical material was identified by Dr. Zefa Valdevina Pereira (Faculty of Biological and Environmental Sciences) and deposited in the herbarium of the Federal University of Grande Dourados-UFGD. Each specimen generated an identification protocol number (DDMS) (Table 1).

\subsection{Bibliographic Survey}

A review of the popular use and phytochemical and pharmacological data of species found in the reserve region of the settlement "April 17" was performed using online journals and books that were published in English, Portuguese and Spanish. The information related to this article was collected from scientific literature databases including Science Direct, PubMed, Scopus and Google Scholar. Articles were selected according to the family, gender, and species (including synonymous species). Due to the widespread use of terms transcribed (traditional use of diseases, symptoms and effects) in review the species were grouped under 12 categories.

\section{Results and Discussion}

For the first time, 89 medicinal plants belonging to 39 families were documented in the study area; the documented plants included trees $(n=60)$, bushes $(n=19)$, and shrubs $(n=1)$ (Table 1). Fabaceae $(n=13)$, 
Myrtaceae $(n=7)$, Rubiaceae $(n=7)$ and Bignoniaceae $(n=5)$ were the most species-rich families (Table 1$)$. The leaves $(41 \%)$, bark $(22 \%)$ and roots $(15 \%)$ were the most commonly reported plant parts for popular use (Table 1). Table 1 presents information on previous scientific research, describing the forms of use of the plants, as well as the useful part of the plant and its use.

These plant species are used for the treatment and prevention of many ailments and due to the widespread use of terms transcribed in review the species were grouped under 10 specific and 2 general categories diseases. The most widespread traditional use, according to the literature review of 10 specific categories diseases of the following plants involves the treatment of gastro-intestinal system diseases (DSD, $44 \mathrm{spp}$ ), followed by the endocrine system diseases (ENM, $24 \mathrm{spp}$ ), infectious diseases (IPD, $20 \mathrm{spp}$ ), musculoskeletal and joint diseases (MCT, $20 \mathrm{spp}$ ), respiratory system diseases (RSD, $19 \mathrm{spp}$ ), skin, eye, ear, nose and oropharynx diseases (SST, 18 spp), obstetrics, gynecology and urinary-tract diseases (GUS, $18 \mathrm{spp}$ ), malignant diseases (NEP, 9 spp), cardiovascular system diseases (CSD, $8 \mathrm{spp}$ ) and central nervous system diseases (NSD, $6 \mathrm{spp}$ ) (Figure 2). Many species have been reported grouped into 2 general categories, as for the treatment inflammatory diseases (ID, 16 spp) and other diseases (OD, $41 \mathrm{spp})$ (Figure 2).

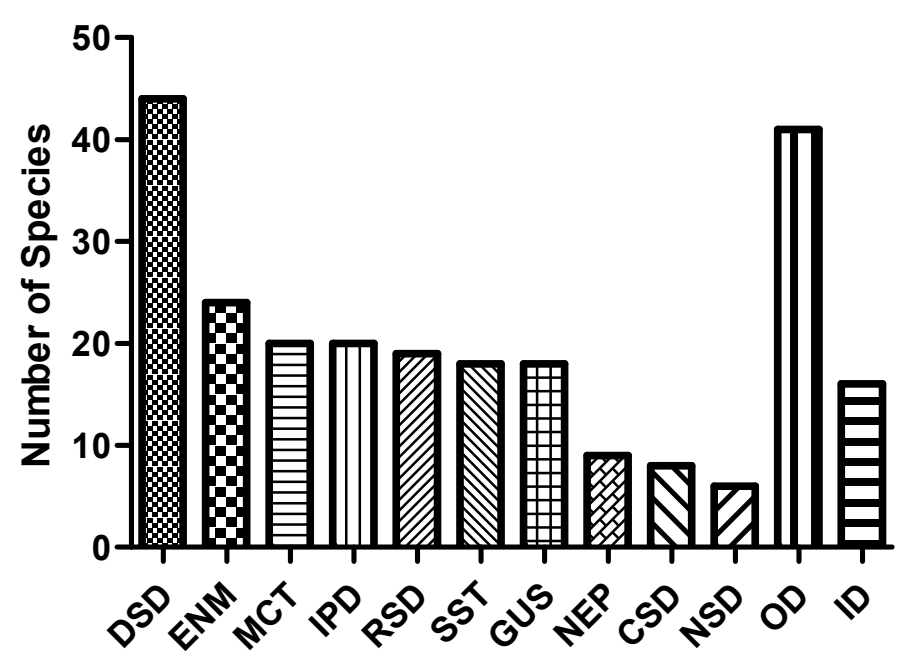

Figure 2. Diseases categories grouped according to a review of species found in the study area. (DSD) Gastro-intestinal System Diseases; (ENM) Endocrine System Diseases; (MCT) Musculoskeletal and Joint Diseases; (IPD) Infectious Diseases; (RSD) Respiratory System Diseases; (SST) Skin, Eye, Ear, Nose and Oropharynx Diseases; (GUS) Obstetrics, Gynecology and Urinary-tract Diseases; (NEP) Malignant Diseases; (CSD) Cardiovascular System Diseases; (NSD) Central Nervous System Diseases; (OD) Others Diseases and (ID) Inflammatory Diseases others

The symptoms and effects mentioned for DSD includes antidiarrheal, carminative, abdominal disorders, dysentery, purgative, laxative, hemorrhoids, hernia, gastritis, ulcer, liver and spleen diseases, flatulent dyspepsia liver abnormalities, gallstones, heartburn, kidney pain and intestinal colic. According to the World Health Organization, gastro-intestinal system diseases (diarrhoeal) are the main cause of death in children under five years old, and there are almost 1.7 billion cases of childhood diarrhoea every year, with approximately 525,000 deaths (Kirk et al., 2017). The OD category include as an example snake bites, aphrodisiac, tonic, emetic, astringent, scurvy, anthelmintic, swelling, malaise and stings insects. 
Table 1. Identification of the species found and recorded in the literature (name, popular use and utilized part) in the reserve area of the settlement "April 17", located in Casa Verde district, Nova Andradina city, MS, Brazil

\begin{tabular}{|c|c|c|c|}
\hline Family & Botanical taxon (DDMS)/Habit & Popular name & Popular uses (Used parts and application) \\
\hline Amaranthaceae & $\begin{array}{l}\text { Gomphrena officinalis Mart. } \\
\text { (4629)/Bush }\end{array}$ & "Para-tudinho", "para-tudo" & $\begin{array}{l}\text { Roots: Tonic, counteracts weakness, general debility \& panacea for } \\
\text { all ills (Almeida et al., 1998; Alzugaray \& Alzugaray, 1983; } \\
\text { Balbach, 1979; Lorenzi \& Matos, 2002) }\end{array}$ \\
\hline \multirow{3}{*}{ Anacardiaceae } & $\begin{array}{l}\text { Anacardium humile A. St. Hil } \\
(5418) / \text { Bush }\end{array}$ & $\begin{array}{l}\text { "Cajuzinho-do-cerrado", "caju do } \\
\text { campo" }\end{array}$ & $\begin{array}{l}\text { Purgative, diarrhoea, anti-inflammatory, astringent \& external } \\
\text { ulcers (Pott \& Pott, 1994; Thomas \& Filho, 1985; Vila Verde et al., } \\
\text { 2003; Agra et al., 2007a) }\end{array}$ \\
\hline & $\begin{array}{l}\text { Astronium fraxinifolium } \text { Schott } \\
(5107) / \text { Tree }\end{array}$ & "Gonçalo-alves" & $\begin{array}{l}\text { Leaves: Allergies, anti-inflammatory, antidiarrhoeal \& ulcers (Viana } \\
\text { et al., 1997, Silva et al., 2011b; Resende et al., 2015) }\end{array}$ \\
\hline & $\begin{array}{l}\text { Tapirira guianensis Aubl. } \\
(6035) / \text { Tree }\end{array}$ & $\begin{array}{l}\text { "Tapirira", "pau-pombo", "cupiúva", } \\
\text { "tatapiririca", jobo, "cedroí," "fresmo" }\end{array}$ & $\begin{array}{l}\text { Leaves: Antibacterial; treatment of malaria, leishmaniasis \& } \\
\text { syphilis (David et al., 1998; Roumy et al., 2009) } \\
\text { Barks: Anti-leishmanial, anti-plasmodial, antibacterial \& } \\
\text { anti-fungal, snake bites, oral thrush \& sore throat (Deharo et al., } \\
\text { 2001; Roumy et al., 2009; Zoghbi et al., 2014; Vásquez et al., 2015) }\end{array}$ \\
\hline \multirow{4}{*}{ Annonaceae } & Annona coriacea $(5818) /$ Tree & "Araticum", "marolo", "araticum-liso" & $\begin{array}{l}\text { Leaves: Stomach diseases, stomatitis, neuralgia, headache, } \\
\text { anti-rheumatic \& anthelmintic (Morais Cardoso et al., 2013); } \\
\text { treatment of chronic diarrhoea (Rodrigues \& Carvalho, 2001) }\end{array}$ \\
\hline & $\begin{array}{l}\text { Annona crassiflora Mart. } \\
(61640) / \text { Tree }\end{array}$ & $\begin{array}{l}\text { "Araticum-do-cerrado", } \\
\text { "araticum-do-campo", } \\
\text { "pinha-do-cerrado", "marolo" }\end{array}$ & $\begin{array}{l}\text { Leaves: Tumours \& analgesic (Roesler et al., 2007) } \\
\text { Seeds: Skin infection \& antidiarrhoeal (Luzia \& Jorge et al., 2013; } \\
\text { Roesler et al., 2006) }\end{array}$ \\
\hline & $\begin{array}{l}\text { Annona dioica A.St.-Hil. } \\
(6037) / \text { Bush }\end{array}$ & "Ceraticum", "arixicum", "ariticum" & $\begin{array}{l}\text { Leaves: Rheumatism, antidiarrhoeal, sedative \& anti-catarrhal (Pott } \\
\& \text { Pott, 1994) }\end{array}$ \\
\hline & $\begin{array}{l}\text { Xylopia aromatica }(\text { Lam.) Mart. } \\
(5937) / \text { Tree }\end{array}$ & "Pimenta de macaco" & $\begin{array}{l}\text { Roots: Anti-malarial (de Mesquita et al., 2007) } \\
\text { Fruits: Carminative \& aphrodisiac (Oliveira et al., 2014) }\end{array}$ \\
\hline \multirow{4}{*}{ Apocynaceae } & $\begin{array}{l}\text { Aspidosperma macrocarpa Mart. } \\
(4835) / \text { Tree }\end{array}$ & No reports & No reports \\
\hline & $\begin{array}{l}\text { Aspidosperma tomentosum Mart. } \\
(3528) / \text { Tree }\end{array}$ & "Guatambu" & $\begin{array}{l}\text { Bark: Hypercholesterolemia, anorexic, diuretic (Silva et al., 2010a; } \\
\text { de Almeida et al., 2019) }\end{array}$ \\
\hline & $\begin{array}{l}\text { Hancornia speciosa Gomes } \\
(5822) / \text { Tree }\end{array}$ & "Mangabeira", "mangaba" & $\begin{array}{l}\text { Leaves: Diabetes, anti-hypertensive \& anti-obesity (Hirschmann \& } \\
\text { Arias, 1990; Rodrigues \& Carvalho, 2001; Macedo \& Ferreira, } \\
\text { 2004; Pereira et al., 2015b) }\end{array}$ \\
\hline & $\begin{array}{l}\text { Himatanthus obovatus (Müll. } \\
\text { Arg.) Woodson }\end{array}$ & “Tiborna”, "pau-de-leite", "janaguba" & Leaves: Cancer, herpes \& worms (Mesquita et al., 2005) \\
\hline Araliaceae & $\begin{array}{l}\text { Schefflera macrocarpa (Cham. \& } \\
\text { Schltdl.) Frodin/Tree }\end{array}$ & $\begin{array}{l}\text { "Mandiocão", "mandiocão-do-campo", } \\
\text { "caixeta", "caixeiteiro" }\end{array}$ & No reports \\
\hline \multirow[t]{2}{*}{ Arecaceae } & $\begin{array}{l}\text { Allagoptera campestris (Mart.) } \\
\text { Kuntze (4793)/Tree }\end{array}$ & $\begin{array}{l}\text { "Buri-do-campo", "pissandó", } \\
\text { "paissandu", "pissandu", } \\
\text { "coqueiro-pissandó" }\end{array}$ & No reports \\
\hline & $\begin{array}{l}\text { Syagrus flexuosa (Mart.) } \\
\text { Becc./Tree }\end{array}$ & $\begin{array}{l}\text { “Coquinho-babão", "acumã”, "coco do } \\
\text { campo" }\end{array}$ & No reports \\
\hline \multirow{4}{*}{ Bignoniaceae } & $\begin{array}{l}\text { Anemopaegma arvense (Vell.) } \\
\text { Stellfeld \& J.F. Souza } \\
(5247) / \text { Shrub }\end{array}$ & $\begin{array}{l}\text { "Vergateza", "vergateso", } \\
\text { "catuaba-do-cerrado", "catuaba", } \\
\text { "pau-de-resposta", } \\
\text { "alecrim-do-campo" }\end{array}$ & $\begin{array}{l}\text { Roots \& leaves: Aphrodisiac, nervous system stimulator, treatment } \\
\text { of inflammation in the ovaries \& varicose veins, stimulant, } \\
\text { insomnia, neurasthenia, nervousness, hypochondria, poor memory, } \\
\text { in recovery from serious illness, asthenia, anxiety, chronic } \\
\text { bronchitis, bronchial asthma \& sexual impotence (Barros, 1982; } \\
\text { Guarim Neto, 1987; Br\&ão, 1991; Lorenzi \& Matos, 2002; } \\
\text { Longhini et al., 2017) }\end{array}$ \\
\hline & $\begin{array}{l}\text { Cybistax antisyphilitica (Mart.) } \\
(4763) / \text { Tree }\end{array}$ & $\begin{array}{l}\text { "Carobinha verde", "caroba de flor } \\
\text { verde", "ipê-mandioca", } \\
\text { "ipê-de-flor-verde", "pé-de-anta", } \\
\text { "cinco-em-folhas", "ipê mirim", } \\
\text { "ipê-amarelo" }\end{array}$ & $\begin{array}{l}\text { Young branches, roots \& leaves: Anti-syphilitic, dysuria, hydrops, } \\
\text { water retention, poultice, syphilitic ulcers, fever, headache \& } \\
\text { invigorating baths (Siqueira, 1982; Guarim Neto, 1987; Sanz-Biset } \\
\text { et al., 2009; Breitbach et al., 2013) } \\
\text { Stem bark: Anti-rheumatic, anti-arthritic, anti-cancer, anti-malarial } \\
\& \text { healing of ulcers (Llorente et al., 2016) }\end{array}$ \\
\hline & $\begin{array}{l}\text { Handroanthus ochraceus } \\
\text { (Cham.) Mattos }\end{array}$ & "Mangabeira", "mangaba" & $\begin{array}{l}\text { Leaves: Diabetes, anti-hypertensive \& obesity prevention } \\
\text { (Hirschmann \& Arias, 1990; Rodrigues \& Carvalho, 2001; Macedo } \\
\text { \& Ferreira, 2004; Pereira et al., 2015b) }\end{array}$ \\
\hline & $\begin{array}{l}\text { Jacaranda decurrens Cham. } \\
(5565) / \mathrm{Bush}\end{array}$ & "Carobinha", "carobinha-do-campo" & $\begin{array}{l}\text { Inflammatory diseases, infections, syphilis, rheumatism, } \\
\text { dermatological diseases, treatment of diarrhoea \& dysentery, blood } \\
\text { cleanser, wound healing in the uterus \& ovary, prostate } \\
\text { inflammation, allergies, diabetes, hyperlipidaemia \& rheumatic } \\
\text { problems (Nunes et al., 2003; Tresvenzol et al., 2007; Gachet \& } \\
\text { Schühly, 2009; Bieski et al., 2012; Neiva et al., 2014) }\end{array}$ \\
\hline
\end{tabular}




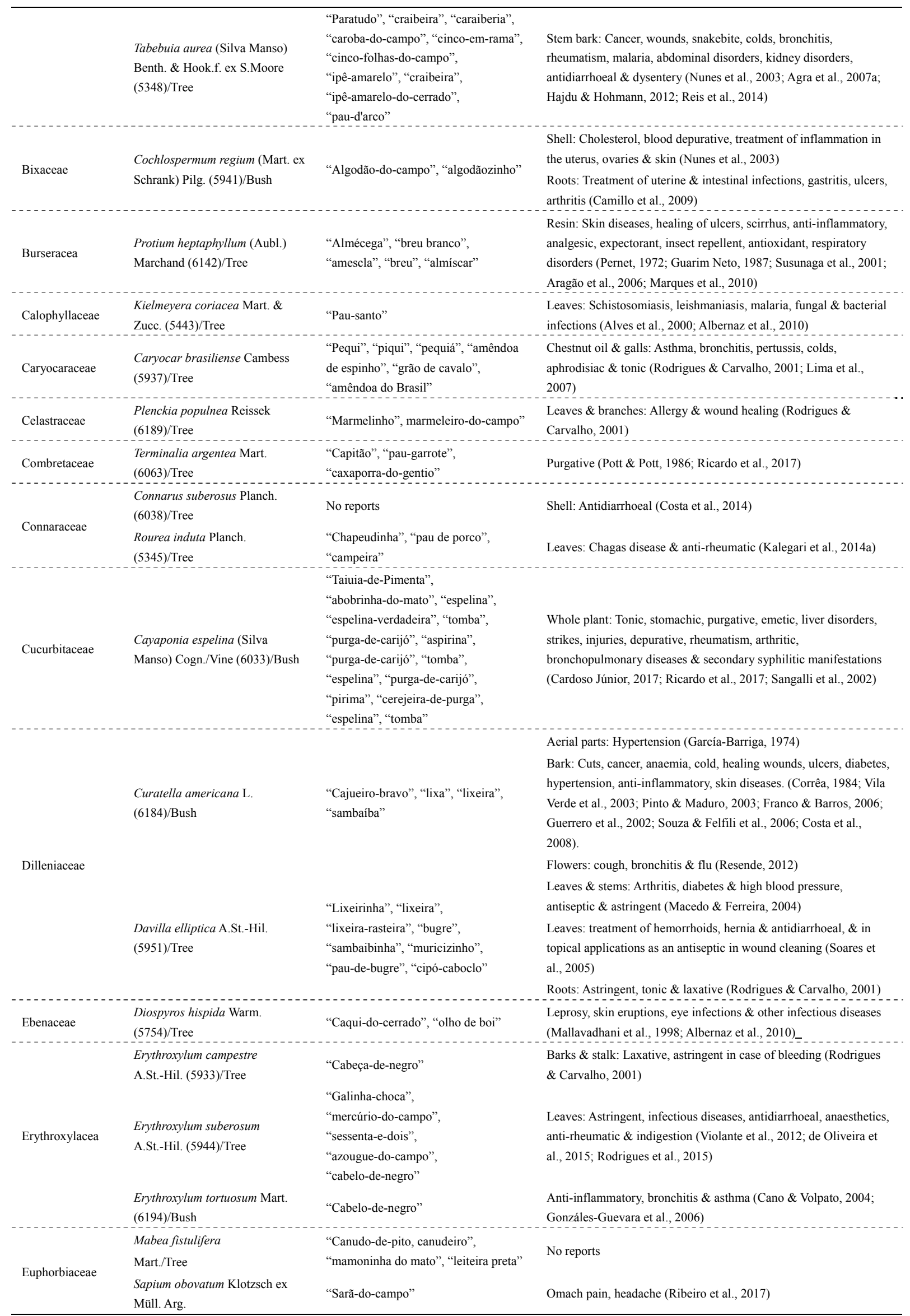


Acosmium subelegans (Mohlenbr.) Yakovlev (5068)/Tree

Anadenanthera peregrina (L.) Speg. (5848)/Tree Andira humilis Mart. ex Benth. (5926)/Tree

Bauhinia rufa (Bong.) Steud. (6029)/Tree

Bowdichia virgilioides Kunth/Tree

Copaifera langsdorffii Desf. (5939)/Tree

Dalbergia miscolobium Benth.

\section{"Caviúna-do-campo"}

Fabaceae

Dimorphandra mollis Benth. (4906)/Tree

Dipteryx alata Vogel (5995)/Tree "Baru"

Diptychandra aurantiaca Tul. (6149)/Tree

Pterodon pubescens (Benth.) (5947)/Tree

Stryphnodendron adstringens (Mart.) Coville/Tree

Vatairea macrocarpa (Benth.) Ducke (6193)/Tree

Aiouea trinervis Meisn. (5924)/Tree

Lauraceae Juss.

Ocotea minarum (Nees \& Mart.) Mez/Tree

"Falso barbatimão", "farinha-seca", "enche-cangalha",

"barbatimão-de-folha-miúda"

"Balsaminho",

"Sucupira-branca", "faveira"

"Barbatimão", "barba-de-timão", "casca-da-virgindade", "faveira", "barbatimão-branco", "barbatimão-verdadeiro"

"Amargoso", "maleiteira", "angelim-do-cerrado"

"Brinco-de-princesa",

"louro-de-Goiás", “uridol”, “urinosa”, Leaves: Aphrodisiac (Moraes, 2005; Maier, 2016) "vergateza"

"Canelinha", "canela vassoura"

C\&idiasis (Rodrigues et al., 2014) CEidiasis (Rodrigues et al., 2014) Shell: Tonic, antipyretic, antimalarial, liver, spleen \& stomach diseases, fever \& malaria (\&rade-Neto et al., 2003; Honório-França et al., 2008; Bonamin et al., 2011) headaches related to digestive disorders (Henrique da Silva et al., 2015; Farmacopeia Brasileira, 2017)

Leaves, roots, bark \& sap: Gastritis, ulcers, bloody diarrhoea, venereal diseases, fever, boil, syphilis, worms, cancer, detoxification, diabetes, obesity, haemorrhoids, swelling, labyrinthitis, pneumonia, tuberculosis, heartburn, liver, indigestion, gallstones, back pain, uterine inflammation, diuretic \& burn (Ribeiro et al., 2017; Pereira et al., 2018)

Lythraceae Lafoensia pacari A.St.-Hil. "Mangava-brava", "pacari", J.St.-Hil. (6049)/Tree "dedaleiro", "louro-da-serra" Trunk bark: Fever \& diabetes (Kassuya et al., 2009; Mori et al., 2011)

\begin{tabular}{|c|c|c|}
\hline $\begin{array}{l}\text { Magnoliaceae } \\
\text { Juss. }\end{array}$ & $\begin{array}{l}\text { Magnolia ovata (A.St.-Hil.) } \\
\text { Spreng. (5954)/Tree }\end{array}$ & "Pinha-do-brejo", "baguaçu" \\
\hline & $\begin{array}{l}\text { Byrsonima basiloba A. Juss. } \\
(6030) / \text { Tree }\end{array}$ & "Murici" \\
\hline & $\begin{array}{l}\text { Byrsonima coccolobifolia Kunth } \\
\text { (6169)/Bush }\end{array}$ & $\begin{array}{l}\text { "Murici", "murici-de-flor-rósea", } \\
\text { "murici-do-cerrado" }\end{array}$ \\
\hline
\end{tabular}
Leaves: Antidiarrhoeal \& gastric ulcer (Figueiredo et al., 2005; Lira et al., 2008)

Antidiarrhoeal (Lorenzi, 2002; Br\&ão et al., 1992)

Leaves: Fever, ulcers, diuretic, asthma \& skin infection(Santos et al., 2019)

Stem bark: Antidiarrhoeal, dysentery, anti-fungal \& anti-inflammatory activity (Rodrigues \& Carvalho, 2001) 


\begin{tabular}{|c|c|c|c|}
\hline & Diplopterys pubipetala (A.Juss.) & & \\
\hline & $\begin{array}{l}\text { W.R. Anderson \& C.C.Davis } \\
(5932) / \text { Bush }\end{array}$ & “Cipó-preto" & No reports \\
\hline \multirow[b]{2}{*}{ Malvaceae Juss. } & $\begin{array}{l}\text { Eriotheca gracilipes (K.Schum.) } \\
\text { A.Robyns }(6185) / \text { Tree }\end{array}$ & "Paineira", "paineira-da-mata" & No reports \\
\hline & $\begin{array}{l}\text { Luehea divaricata } \text { Mart. \& Zucc. } \\
(6046) / \text { Tree }\end{array}$ & "Açoita cavalo", "caiboti" & $\begin{array}{l}\text { Leaves: Uric acid build-up, kidney disease, throat inflammation, } \\
\text { influenza, haemorrhoids, pneumonia, muscle aches, cough \& } \\
\text { tumours (Tirloni et al., 2018) }\end{array}$ \\
\hline Melastomataceae & $\begin{array}{l}\text { Miconia albicans }(\mathrm{Sw} .) \text { Triana } \\
(5492) / \mathrm{Bush}\end{array}$ & "Canela-de-velho" & $\begin{array}{l}\text { Leaves: Anti-inflammatory \& diabetes prevention (Lima et al., } \\
2018\end{array}$ \\
\hline Moraceae & $\begin{array}{l}\text { Brosimum gaudichaudii trécul } \\
(5406) / \text { Tree }\end{array}$ & $\begin{array}{l}\text { "Mama-de-cadela", "mama-cadela", } \\
\text { "mamica-de-cadela", "algodãozinho", } \\
\text { "inharé", "mama-cachorro" }\end{array}$ & $\begin{array}{l}\text { Stem bark, leaves, roots \& latex: Vitiligo, skin diseases, infection, } \\
\text { venereal disease, furuncle, "impingem" (superficial skin mycoses), } \\
\text { cancer, anaemia, heart disease, pneumonia, burning skin, tonic, } \\
\text { anti-inflammatory, rheumatism, kidney disease, wound healing, flu, } \\
\text { bronchitis, detoxification, improvement of blood circulation, } \\
\text { general pain, \& mosquito bite allergy (Rodrigues \& Carvalho, } \\
2001 \text {; Amorozo, 2002; Agra et al., 2008; Monteiro et al., 2014; } \\
\text { Ribeiro et al., 2017) }\end{array}$ \\
\hline \multirow{7}{*}{ Myrtaceae } & $\begin{array}{l}\text { Campomanesia adamantium } \\
\text { (Cambess.) O.Berg. (5856)/Tree }\end{array}$ & "Guavira" & $\begin{array}{l}\text { Leaves \& fruits: Anti-inflammatory, antidiarrhoeal, urinary } \\
\text { infection, anti-depressant, anti-hyperalgesic, rheumatism, } \\
\text { hypocholesterolaemia, treatment of cystitis \& urethritis, antiseptic } \\
\& \text { stomach disorders (Lorenzi, 2000; Piva, 2002; Ramos et al., } \\
\text { 2007; Coutinho et al., 2008; Lorenzi et al., 2008; Vieira et al., 2011; } \\
\text { Pascoal et al., 2014; Lescano et al., 2016; Souza et al., 2017) } \\
\text { Roots: Diabetes (Alice et al., 1995) }\end{array}$ \\
\hline & Eugenia aurata $\mathrm{O}$. Berg & $\begin{array}{l}\text { "Murtinha", "pitangobí", } \\
\text { "pitangobí-azul-do-cerrado" }\end{array}$ & No reports \\
\hline & $\begin{array}{l}\text { Eugenia dysenterica } \text { (Mart.) DC. } \\
(6425) / \text { Tree }\end{array}$ & "Cagaita", "cagaiteira" & $\begin{array}{l}\text { Leaves: Antidiarrhoeal, diabetes, jaundice, kidney disease, bladder } \\
\text { infections, laxative \& cardiovascular diseases (Almeida et al., 1998; } \\
\text { Martinotto et al., 2007; Silva et al., 2010a; Lima et al., 2010) }\end{array}$ \\
\hline & $\begin{array}{l}\text { Eugenia pitanga }(\mathrm{O} . \mathrm{Berg}) \\
\text { Kiaersk. }(6111)\end{array}$ & No reports & No reports \\
\hline & $\begin{array}{l}\text { Eugenia punicifolia (Kunth) DC. } \\
(5925) / \text { Bush }\end{array}$ & $\begin{array}{l}\text { "Pitanga do campo", "murta } \\
\text { vermelha", "pedra-ume caá", } \\
\text { "pitanga-do-cerrado", "muta" }\end{array}$ & $\begin{array}{l}\text { Leaves \& fruits: Diabetes prevention, cough, antidiarrhoeal, } \\
\text { stomach disorders, pain, inflammation, fever, influenza, sores \& } \\
\text { infections (Brunetti et al., 2006; Leite et al., 2010; Rocha et al., } \\
\text { 2011; Pascual et al., 2011; Sales et al., 2014; Basting et al., 2014; } \\
\text { Costa et al., 2016) }\end{array}$ \\
\hline & Myrcia bella Cambess./Bush & "Pedra-ume caá", "mercurinho" & $\begin{array}{l}\text { Leaves: Astringent, diabetes, antidiarrhoeal, diuretic, coagulant, } \\
\text { hypertension \& ulcers (Saldanha et al., 2013; Vareda et al., 2014; } \\
\text { Serpeloni et al., 2015) }\end{array}$ \\
\hline & Myrcia guianensis (Aubl.) DC. & "Pitanga-miúda", "pedra-ume-caá" & No reports \\
\hline Ochnaceae & Ouratea spectabilis (Mart.) Engl. & "Folha-de-serra" & Gastric \& rheumatic disorders (Paulo et al., 1986) \\
\hline Primulaceae & Myrsine umbellata Mart./Tree & "Capororoca", "pororoca" & Snake bites, tumours \& wounds (Rodrigues \& Carvalho, 2001) \\
\hline Proteaceae Juss. & $\begin{array}{l}\text { Roupala montana Aubl. } \\
(6049) / \text { Tree }\end{array}$ & $\begin{array}{l}\text { "Carvalho brasileiro", "carne de vaca", } \\
\text { "congonha", "caxuá", "farinha-seca" }\end{array}$ & $\begin{array}{l}\text { Leaves: Pain in the kidneys, legs \& spine; malaise; \& soothing pain } \\
\text { (Souza et al., 2014) } \\
\text { Bark: Fever \& pain in the stomach (Júnior \& Júnior, 2009) }\end{array}$ \\
\hline \multirow{7}{*}{ Rubiaceae Juss. } & Alibertia edulis (Rich.) A.Rich. & $\begin{array}{l}\text { "Marmelada-bola", } \\
\text { "marmelo-do-cerrado" }\end{array}$ & Hypertension (de Santana Aquino et al., 2017) \\
\hline & $\begin{array}{l}\text { Cordiera sessilis (Vell.) Kuntze } \\
(6145) / \text { Bush }\end{array}$ & $\begin{array}{l}\text { "Marmelinho", } \\
\text { "marmelada-de-cachorro" }\end{array}$ & Leaves: Skin diseases (Rodrigues \& Carvalho, 2001) \\
\hline & $\begin{array}{l}\text { Guettarda viburnoides Cham. \& } \\
\text { Schltdl. (6416)/Tree }\end{array}$ & "Veludo branco" & $\begin{array}{l}\text { Stalk: Anti-inflammatory, antidiarrhoeal, respiratory diseases, } \\
\text { rheumatism, fever, diabetes \& hepatitis (Capasso et al., 1998; } \\
\text { Coelho-Ferreira, 2009; Magalhães et al., 2019) }\end{array}$ \\
\hline & $\begin{array}{l}\text { Palicourea coriacea (Cham.) } \\
\text { K.Schum. (5928)/Bush }\end{array}$ & $\begin{array}{l}\text { "Douradinha do campo", "congonha } \\
\text { do campo", "douradinha" }\end{array}$ & $\begin{array}{l}\text { Roots \& leaves: Liver diseases, diuretic, renal calculi, kidney \& } \\
\text { bladder infections \& pain (Laureano, 2001; Nunes et al., 2003; } \\
\text { Freitas et al., 2011) }\end{array}$ \\
\hline & $\begin{array}{l}\text { Psychotria poeppigiana Müll. } \\
\text { Arg. (5292)/Bush }\end{array}$ & $\begin{array}{l}\text { "Arbusto da boca dolorida", "lábios de } \\
\text { fogo", "beijo", "beijo de negra", } \\
\text { "chapéu do diabo" }\end{array}$ & $\begin{array}{l}\text { Leaves: Gastrointestinal disorders, pain, stomach pain, dyspnoea, } \\
\text { anti-inflammatory for bites \& stings (snakes, insects \& scorpions), } \\
\text { fever, infection, diabetes, oral abortifacient, wounds \& rashes, cuts } \\
\& \text { bleeding (Coe \& \&erson, 1996; Taylor et al., 2006; Pino-Benítez, } \\
\text { 2006; Guerrero et al., 2010) }\end{array}$ \\
\hline & $\begin{array}{l}\text { Tocoyena brasiliensis Mart. } \\
(6174) / \text { Tree }\end{array}$ & "Jenipapinho" & Burn \& rheumatism (Souza et al., 2013) \\
\hline & $\begin{array}{l}\text { Tocoyena formosa (Cham. \& } \\
\text { Schltdl.) K.Schum. (6202)/Tree }\end{array}$ & "Jenipapo do bravo" & $\begin{array}{l}\text { Coughs, torsion, cystitis, rheumatism, \& renal \& cardiac problems } \\
\text { (de Albuquerque et al., 2007) }\end{array}$ \\
\hline
\end{tabular}




\begin{tabular}{|c|c|c|c|}
\hline Salicaceae & $\begin{array}{l}\text { Casearia sylvestris Sw. } \\
(6411) / \text { Tree }\end{array}$ & $\begin{array}{l}\text { "Guaçatonga", "guafatonga", } \\
\text { "erva-de-lagarto", "lingua-de-tiu", } \\
\text { "cafezinho-do-mato", "corta-lengua", } \\
\text { "erva-de-bugre", "café selvagem" }\end{array}$ & $\begin{array}{l}\text { Leaves: Diarrhoea, fever, toxicity, rheumatism, skin conditions \& } \\
\text { snake bites (Ferreira et al., 2011) } \\
\text { Stem bark: amti-inflammatory (Ferreira et al., 2016) }\end{array}$ \\
\hline Sapindaceae & $\begin{array}{l}\text { Allophylus edulis (A.St.-Hil. et } \\
\text { al.) Hieron. (3508)/Tree }\end{array}$ & $\begin{array}{l}\text { "Chal-chal", "vacum", } \\
\text { "fruto-de-pombo", "vacunzeiro", } \\
\text { "murta vermelha", "pau-de-pedreira", } \\
\text { "baga-de-morcego" }\end{array}$ & $\begin{array}{l}\text { Leaves: Diabetes, anti-inflammatory, intestinal \& digestive } \\
\text { problems, high blood pressure, wound washing, liver \& digestive } \\
\text { troubles, cholecystitis \& jaundice (Arisawa et al., 1989; Körbes, } \\
\text { 1995; Franco \& Fontana, 2001; Díaz et al., 2008) }\end{array}$ \\
\hline Sapotaceae & $\begin{array}{l}\text { Pouteria torta (Mart.) Radlk. } \\
(6414) / \text { Tree }\end{array}$ & $\begin{array}{l}\text { "Guapeva", "curiola", "acá ferro", } \\
\text { "abiu do cerrado", "grão de galo" }\end{array}$ & Bark: Dysentery (Costa et al., 2014) \\
\hline $\begin{array}{l}\text { Solanaceae } \\
\text { A.Juss. }\end{array}$ & $\begin{array}{l}\text { Solanum lycocarpum A.St.-Hil. } \\
(5955) / \text { Tree }\end{array}$ & $\begin{array}{l}\text { "Fruta do lobo", "lobeira", } \\
\text { "jurubebão", "beringela-do-cerrado" }\end{array}$ & $\begin{array}{l}\text { Roots \& green fruits: Sedative, epilepsy, spasms, abdominal \& } \\
\text { renal pain, haemorrhoids, influenza, hepatitis, diabetes, obesity, } \\
\text { snakebite, \& tissue atrophy (Munari et al., 2012) } \\
\text { Fruits: Fruits: Hypoglycaemic \& cholesterol lowering (Schwarz et } \\
\text { al., 2007) } \\
\text { Leaves: Cough \& malaria (Ribeiro et al., 2017) }\end{array}$ \\
\hline \multirow{4}{*}{$\begin{array}{l}\text { Vochysiaceae } \\
\text { A.St.-Hil. }\end{array}$} & $\begin{array}{l}\text { Qualea grandiflora Mart. } \\
(6171) / \text { Tree }\end{array}$ & "Cinzeiro", "boizinho", "pau-terrinha" & $\begin{array}{l}\text { Leaves \& bark: Treatment of bloody diarrhoea, intestinal colic, } \\
\text { amoebiasis, skin diseases, inflammation, ulcers \& gastritis (de } \\
\text { Mesquita et al., 2015) }\end{array}$ \\
\hline & $\begin{array}{l}\text { Qualea multiflora Mart. } \\
(6178) / \text { Tree }\end{array}$ & $\begin{array}{l}\text { "Cinzeiro", "pau-tucano", } \\
\text { "uva-puva-do-campo", } \\
\text { "pau-terra-do-campo", "pau-terra-liso" }\end{array}$ & $\begin{array}{l}\text { Bark: Antidiarrhoeal, ulcers, gastric diseases \& anti-inflammatory } \\
\text { (Santos et al., 2011) }\end{array}$ \\
\hline & $\begin{array}{l}\text { Qualea parviflora } \text { Mart. } \\
(6026) / \text { Tree }\end{array}$ & $\begin{array}{l}\text { "Pau-terra", "pau-ferro", } \\
\text { "pau-de-tucano" }\end{array}$ & $\begin{array}{l}\text { Leaves \& bark: Treatment of bloody diarrhoea, intestinal colic, } \\
\text { amoebiasis, skin diseases, ulcers \& gastritis (de Mesquita et al., } \\
\text { 2015) }\end{array}$ \\
\hline & Vochysia tucanorum Mart./Tree & $\begin{array}{l}\text { "Pau-de-tucano", "tucaneiro", } \\
\text { "vinheiro". }\end{array}$ & No reports \\
\hline
\end{tabular}

\subsection{Pharmacological and Phytochemical Studies}

Many of the species found in the study area have already been chemically and biologically evaluated, possibly in an attempt to prove their popular indication. Table 2 comprises a scientific survey reporting the chemical and pharmacological studies that have already been carried out on the target species. Regarding pharmacological studies, only Gomphrena officinalis, Schefflera macrocarpa, Allagoptera campestris, Syagrus flexuosa, Cayaponia espelina, Sapium obovatum, Diptychandra aurantiaca, Diplopterys pubipetala, Eugenia pitanga, Myrsine umbellate, Anadenanthera peregrina, Andira humilis, Dalbergia miscolobium, Eriotheca gracilipes and Tocoyena brasiliensis have not been studied (Table 2).

Scientific evidence has shown that most studies are related to the main popular uses. Anacardium humile, Astronium fraxinifolium, Cochlospermum regium, Protium heptaphyllum, Curatella americana, Davilla elliptica, Lafoensia pacari, Byrsonima basiloba, Byrsonima intermedia, Eugenia dysenterica, E. punicifolia, Qualea grandiflora and $Q$. parviflora are grouped gastro-intestinal system diseases category and have associated scientific studies that validate their effects on some classes of intestinal disorders, mainly diarrhoea and gastrointestinal conditions (Table 1,2).

Another prominent category is infectious diseases, among which some species, such as Tapirira guianensis, Annona coriacea, A. crassiflora, Xylopia aromatica, Himatanthus obovatus, Jacaranda decurrens, Cochlospermum regium, Kielmeyera coriacea, Diospyros hispida, Erythroxylum suberosum, Stryphnodendron adstringens, Ocotea minarum, Strychnos pseudoquina, Byrsonima intermedia, Brosimum gaudichaudii, Campomanesia adamantium and Eugenia dysenterica, have popular indications and associated studies that prove their effects (Table 1,2). These data show high herbal diversity among medicinal plants in the reserve area of the settlement "April 17", located in Casa Verde district, Nova Andradina city, MS, Brazil, as well as great potential for further studies on therapeutic activities. Moreover, the conservation of biodiversity ensures the sustainability of natural resources and allows the maintenance of various services essential to human well-being.

In the review, only twelve species were not chemically studied, and fourteen did not have associated pharmacological studies. Among the studied species for which contents were isolated, the predominant class of substances was terpenes, followed by phenolic compounds and alkaloids (Table 2). 
Table 2. Pharmacological and chemical studies of the species found in the reserve area of the settlement "April 17", located in Casa Verde district, Nova Andradina city.

\begin{tabular}{|c|c|c|}
\hline Species & Pharmacological studies & Chemical studies \\
\hline Gomphrena officinalis Mart. & No reports & No reports \\
\hline Anacardium humile A. St. Hil. & $\begin{array}{l}\text { Larvicidal (Porto et al., 2008); gastric lesion reduction (Luiz-Ferreira } \\
\text { et al., 2008; Luiz-Ferreira et al., 2010); anthelmintic (Nery et al., } \\
\text { 2010); anti-microbial (Pereira et al., 2011; Perim et al., 2018) \& } \\
\text { anti-hypoglycaemic effects (Urzêda et al., 2013) }\end{array}$ & $\begin{array}{l}\text { Tannins, flavonoids, terpenes, coumarins, saponins (Agra } \\
\text { et al., 2007a); tannins, flavonoids, alkaloids (Nery et al., } \\
\text { 2010); flavonoids (Luiz-Ferreira et al., 2010); phenolic } \\
\text { compounds, catechins \& terpenes (Cecílio et al., 2012) }\end{array}$ \\
\hline Astronium fraxinifolium Schott. & $\begin{array}{l}\text { Antiviral (Cecílio et al., 2012); antibacterial (Montanari et al., 2012); } \\
\text { gastroprotective \& antioxidant effects (Martins et al., 2017) }\end{array}$ & Terpenes (Montanari et al., 2012) \\
\hline Tapirira guianensis Aubl. & $\begin{array}{l}\text { Cytotoxic (David et al., 1998; Taylor et al., 2013); anti-protozoal, } \\
\text { antibacterial, anti-fungicidal (Roumy et al., 2009); anti-proliferative } \\
\text { (Silva-Oliveira et al., 2016); vasodilatory \& antioxidant effects } \\
\text { (Rodrigues et al., 2017) }\end{array}$ & $\begin{array}{l}\text { Terpenoids (Correia et al., 2003, 2008; Zoghbi et al., 2014; } \\
\text { Silva-Oliveira et al., 2016); flavonoids (Silva-Oliveira et } \\
\text { al., 2016; Rodrigues et al., 2017); norisoprenoids } \\
\text { (Silva-Oliveira et al., 2016); tannins (Rodrigues et al., } \\
\text { 2017); flavonoids \& phenolic acids (Martins et al., 2017) }\end{array}$ \\
\hline Annona coriacea Mart. & $\begin{array}{l}\text { Insecticidal (Coelho et al., 2007; Costa et al., 2012; Freitas et al., } \\
\text { 2014); phytotoxic (Formagio et al., 2010; Novaes et al., 2016); } \\
\text { anti-protozoal (Toledo et al., 2011; Siqueira et al., 2011); antioxidant } \\
\text { (Benites et al. 2015; Novaes et al., 2019); anti-proliferative, enzymatic } \\
\text { inhibitory (Formagio et al., 2015); cytoprotective (Júnior et al., 2016); } \\
\text { anti-tumour (Tundis et al., 2017; Gomes et al., 2019); anti-fungal } \\
\text { (Almeida-Apolonio et al., 2019); anxiolytic \& anti-depressant effects }\end{array}$ & $\begin{array}{l}\text { Terpenes (Mussini et al., 1973; Siqueira et al., 2011); } \\
\text { acetogenins (Yu et al., 1994; Silva et al, 1996; Silva et al., } \\
\text { 1997; Silva et al., 1998); alkaloids (Machado et al., 2013); } \\
\text { phenolic compounds (Freitas et al., 2014; Júnior et al., } \\
\text { 2016; Novaes et al., 2018; Novaes et al., 2019; Monteiro et } \\
\text { al., 2020) \& tannins (Benites et al. 2015) }\end{array}$ \\
\hline
\end{tabular}

(Monteiro et al., 2020)

Antioxidant (Santos et al., 1996; Roesler et al., 2006, 2011; de Souza

et al., 2012; Justino et al., 2016); cytotoxic (Santos et al., 1996); larvicidal (Pimenta et al., 2003); antimutagenic (Vilar et al., 2008); anti-arthritic, anti-plasmodial (de Mesquita et al., 2007); antibacterial (Silva et al., 2014); anti-glycant (Justino et al., 2016) \& pancreatic lipase inhibitive effects (Pereira et al., 2017)

Anti-inflammatory, anti-hypoglycaemic, anti-proliferative \& antioxidant effects (Formagio et al., 2013)

Annona dioica A.St.-Hil Anti-malarial (Garavito et al., 2006); larvicidal (Rodrigues et al., 2006); anti-plasmodial (de Mesquita et al., 2007); cytotoxic

Xylopia aromatica (Lam.) Mart. (Suffredini et al., 2007; Taylor et al., 2013); anti-tumour, anti-protease (Peter et al., 2008) \& anti-inflammatory effect (Oliveira et al., 2014)

Aspidosperma macrocarpa Mart. Antioxidant effect (Silva et al., 2009)

Anti-nociceptive \& anti-inflammatory effects (Aquino et al., 2013a) Anti-hypertensive (Ferreira et al, 2007a; Ferreira et al 2007b);

Aspidosperma tomentosum Mart. chemopreventive (Endringer et al., 2009b); antioxidant \&

Hancornia speciosa Gomes anti-inflammatory effects (Endringer et al., 2009a; Silva et al., 2011a)

Himatanthus obovatus (Müll. Arg.) Anti-tumour \& anti-microbial effects (Mesquita et al., 2009; Toledo et

Woodson al., 2011)

Schefflera macrocarpa (Cham. \&

Schltdl.) Frodin

No reports

Allagoptera campestris (Mart.)

Kuntze

No reports

No reports

Syagrus flexuosa (Mart.) Becc.

Anemopaegma arvense (Vell.)

Stellfeld \& J.F. Souza

Antioxidant (Tabanca et al., 2007); anti-plasmodial (de Mesquita et

al., 2007) \& anti-fungal effects (Costanzo et al., 2013)

Cybistax antisyphilitica. (Mart.)

Mart.

Handroanthus ochraceus (Cham.)

Mattos

Activity against Aedes aegypti larvae (Rodrigues et al., 2005)

Cytotoxic effects (Correia et al., 2016)

Cytotoxic (Subbaramaiah et al., 2000; Casagr\&e et al., 2014); antioxidant (Carvalho et al., 2009); anti-microbial, chemopreventive effects (Zatta et al., 2009); effects on the development of the

Jacaranda decurrens Cham. reproductive system in male rats (Arena et al., 2012); anti-inflammatory (Santos et al., 2012a) \& anti-obesity effects (Antunes et al., 2016)

Tabebuia aurea (Silva Manso) Anti-microbial (Barbosa-Filho et al., 2004a; Santos et al., 2015);

Benth. \& Hook.f. ex S. Moore anti-inflammatory, myotoxic, anti-haemorrhagic (Reis et al., 2014) \& Phenolic acids \& terpenes (Barbosa-Filho et al., 2004a) anti-dematogenic effects (Santos et al., 2015)
No reports

Phytosterols, tocopherols \& unsaturated fatty acids (de Mesquita et al., 2007); flavonoids, isoflavones, carotenoids (Justino et al., 2016) \& alkaloids (Pereira et al., 2017)

Flavonoids (de Souza et al., 2012; Formagio et al., 2013) \& alkaloids (Justino et al., 2016)

Terpenes (Martins et al., 1998); phenolic acids (de Souza et al., 2012) \& flavonoids (Oliveira et al., 2014)

Indole alkaloid (Bolzani et al., 1987)

dole alkaloids (Pereira et al., 2007; Dolabelas

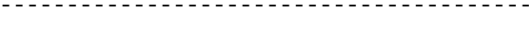

Terpenoids, steroids \& tannins (Honda et al., 1990; Br\&ão et al., 2010)

Iridoides (Lima, 2005)

No reports

No reports

Flavanolignans (Tabanca et al., 2007) \& flavonoids

(Costanzo et al., 2013)

Iridoids (Felicio et al., 1994) \& quinones (Rodrigues et al., 2005)

Glycerides, carboxylic acids, phytosteroid \& terpenoids (Salatino et al., 2020)

Triterpenes (Var\&a et al., 1992; Subbaramaiah et al., 2000; Carvalho et al., 2009) \& flavonoids (Blatt et al., 1998; Antunes et al., 2016) 
Cytotoxic (Ceschini \& Campos, 2006; Taylor et al., 2013);

Cochlospermum regium (Mart. ex Schrank) Pilg.

_...

Protium heptaphyllum (Aubl.) Marchand

Kielmeyera coriacea Mart. \& Zucc.

Caryocar brasiliense Cambess.
Terminalia argentea Mart.
Rourea induta Planch.
Cayaponia espelina (Silva Manso)
Cogn.

Curatella americana $\mathrm{L}$.

Anti-inflammatory, peripheral analgesic (Alex\&re-Moreira et al., et al., 2009); genotoxic (Vilar et al., 2009); anti-fungal (Toledo et al., 2015); antioxidant, hypolipidaemic (Lopes et al., 2016) \& antioxidant effects (Fujishima et al., 2018; Nunes et al., 2018)

Modulatory (Carlos et al., 2005); anti-microbial (Michelin et al., 2005); anti-mycobacterial (Lopes et al., 2007); anti-nociceptive,

Davilla elliptica A.St.-Hil. anti-dematogenic, gastroprotective (Azevedo et al., 2007; Campos et al., 2013; Kushima et al., 2009); immunomodulatory, anti-Helicobacter pylori (Kushima et al., 2009); anti-tumour (Carli et al., 2009) \& mutagenic effects (Biso et al., 2010)

Diospyros hispida Warm. Anti-microbial \& anti-fungal effects (Gu et al., 2004; Albernaz et al., 2010)

Erythroxylum campestre A.St.-Hil. Anti-tumour effects (Pereira et al., 2015a)

Lethality against Artemia salina (do Nascimento et al., 2012a);

Erythroxylum suberosum A.St.-Hil. anti-microbial, antioxidant, cytotoxic (Violante et al., 2012); anti-rheumatic \& anti-inflammatory effects (de Oliveira et al., 2015)

\section{Erythroxylum tortuosum Mart.} Anti-inflammatory, anti-bronchitis \& anti-asthma effects (Cano \& Volpato, 2004; Gonzáles-Guevara et al., 2006)

\section{Mabea fistulifera Mart.}

Antioxidant \& anti-inflammatory effects (Coqueiro, 2006)

Sapium obovatum Klotzsch ex Müll.Arg.

$\&$ anti-fungal effects (Carvalho et al., 2018)

(Susunaga, 1996); anti-inflammatory-related (Siani et al., 1999); et al., 2017; de Melo et al., 2019); anti-mutagenic (de Lima et al., Anti-ulcerogenic, anti-protozoal, anti-fungal (Garcia Cortez et al., et al., 2004; Biesdorf, et al., 2012); stimulation of oxygen glycogenolysis \& glycolysis (Zagoto et al., 2006); schistosomicidic (Sela et al., 2010; de Mesquita et$$
\text { (Biesdorf, et al., 2012) }
$$

markers \& blood pressure reduction (Mir\&a-Vilela et al., 2009);

2018): endothelial vasorelaxation (Oliveira et al., 2018) \&

Haemolytical (Kalegari et al., 2011); antibacterial, antioxidant,

hepatoprotective (Kalegari et al., 2014a) \& anti-nociceptive effects
Phenolic acids, flavonoids \& condensed tannins (Mir\&a Pedroso et al., 2019)

Terpenes (Zoghbi et al., 1995; Susunaga, 1996; Maia et al., 2000; Susunaga et al., 2001; Oliveira et al., 2004a; Oliveira et al., 2005; Vieira Júnior et al., 2005; B\&eira et al., 2006; Marques et al., 2010; Santos et al., 2012b; de Lima et al., 2016; Mobin et al., 2017)

$-$

Xanthones (Mesquita et al., 1987; Garcia Cortez et al., 1998; Cortez et al., 2002; Martins et al., 2006; Zagoto et al., 2006) \& triterpenes (Biesdorf, et al., 2012)

Phenolic acids (Mir\&a-Vilela et al., 2009; Rocha et al.,

2015); fatty acids \& carotenoids (Moreira et al., 2019)

Triterpenes (de Souza et al., 1990; Espindola et al., 2018)

Triterpenoids, lignan, flavonoids (Garcez et al., 2003); flavonoids, saponins, \& phytosterols (Beserra et al., 2018)

Benzoquinones (Costa et al., 2014)

Flavonoids (Kalegari et al., 2011; Kalegari et al., 2014b)

\section{No reports}

Phenolic compounds (El-Azizi et al., 1980; Lopes et al., 2016); flavonoids, saponins, terpenes, tannins (Costa et al., 2008) \& proanthocyanidins (Hiruma-Lima et al., 2009)

Flavonic, saponin heteroside compounds, steroids, coumarins, tannins, steroids, triterpenes (Michelin et al., 2005; Soares et al., 2005; Biso et al., 2010); flavonoids (Carlos et al., 2005; Michelin et al., 2005; Biso et al., 2010)

$\&$ derivatives of phenolic acids (Biso et al., 2010)

Terpenes (Ganapaty et al., 2005) \& alkaloids (Aynilian et al., 1974; Pereira et al., 2015b) Flavonoids (Bohm et al., 1988) Phenolic compounds, alkaloids (Bohm et al., 1988); diterpenes, flavonoids (Rodrigues et al., 2015); flavonoids, tannins, coumarins, saponins (Rodrigues et al., 2015); triterpenes, alkaloids, anthocyanins, coumarins, flavonoids \& condensed tannins (de Oliveira et al., 2015)

Tannins (Ishino et al., 2012)

Flavonoids (Kinghorn, 2001) \& flavanone glycosides (Coqueiro et al., 2007)

No reports 


\begin{tabular}{|c|c|c|}
\hline $\begin{array}{l}\text { Acosmium subelegans (Mohlenbr.) } \\
\text { Yakovlev }\end{array}$ & Anti-convulsant effects (Sousa et al., 2009) & Alkaloids (Oliveira et al., 1994) \\
\hline $\begin{array}{l}\text { Anadenanthera peregrina }(\mathrm{L} .) \\
\text { Speg. }\end{array}$ & No reports & $\begin{array}{l}\text { Phenolic compounds (Carneiro et al., 2012; Sartori et al., } \\
\text { 2014; Mota et al., 2017) }\end{array}$ \\
\hline Andira humilis Mart. ex Benth. & No reports & Phenolic compounds (Garcez et al., 2010) \\
\hline Bauhinia rufa (Bong.) Steud. & $\begin{array}{l}\text { Proteinase inhibitory (Nakahata et al., 2001; Vilela \& Sampaio, 2008); } \\
\text { anti-fungal (Duarte-Almeida et al., 2004; Correia et al., 2016) \& } \\
\text { thrombolytic effects (Silveira et al., 2016) }\end{array}$ & Terpenes (Duarte-Almeida et al., 2004) \\
\hline Bowdichia virgilioides Kunth & $\begin{array}{l}\text { Cytotoxic (Torrenegra et al., 1989); anti-malarial (Deharo et al., } \\
\text { 2001); anti-microbial (Almeida et al., 2006; Agra et al., 2013); } \\
\text { anti-nociceptive, anti-inflammatory (Barros et al., 2010; Thomazzi et } \\
\text { al., 2010; Silva et al., 2010b); antibacterial (Leite et al., 2014a); } \\
\text { muscular damage \& oxidative stress protective (dos Santos et al., } \\
\text { 2014); larvicidal (Bezerra-Silva et al., 2015) \& anti-hyperglycaemic } \\
\text { effects (Silva et al., 2015) }\end{array}$ & $\begin{array}{l}\text { Terpenes (Arriaga et al., 1998; Cordero et al., 2004); } \\
\text { tannins, flavonoids (Arriaga et al., 2000; Leite et al., } \\
\text { 2014a) \& alkaloids (Torrenegra et al., 1989; Barbosa-Filho } \\
\text { et al., 2004b) }\end{array}$ \\
\hline Copaifera langsdorffii Desf. & $\begin{array}{l}\text { Gastroprotective (Paiva et al., 2004, Motta et al., 2018); } \\
\text { ischaemia/reperfusion of intestinal tissue protective (Paiva et al., } \\
\text { 2004); anti-fungal (Amorim et al., 2004; Zimmermam-Franco et al., } \\
\text { 2013); larvicidal (Mendonça et al., 2005); immunomodulatory } \\
\text { (Rosario et al., 2008); antibacterial (Souza et al., 2011); } \\
\text { anti-inflammatory (Gelmini et al., 2013); chemopreventive (Senedese } \\
\text { et al., 2013); anti-psychotic (Gelmini et al., 2013); antioxidant } \\
\text { (Gelmini et al., 2013; Batista et al., 2016); antibacterial (Bonan et al., } \\
\text { 2015); apoptotic (Cardoso et al., 2017) \& colon carcinogenesis } \\
\text { protective effects (Cardoso et al., 2017; Tobouti et al., 2017) }\end{array}$ & $\begin{array}{l}\text { Terpenes, xyloglucans \& phenolic compounds (Rosario et } \\
\text { al., 2008; do Nascimento et al., 2012b; Gelmini et al., } \\
\text { 2013; Senedese et al., 2013; Baldissera et al., 2014; } \\
\text { Nogueira et al., 2015) }\end{array}$ \\
\hline Dalbergia miscolobium Benth. & No reports & $\begin{array}{l}\text { Phenolic compounds (Gregson et al., 1978; Vasudeva et al., } \\
\text { 2009; Kite et al., 2010) \& triterpenoids (Salatino et al., } \\
\text { 2020) }\end{array}$ \\
\hline Dimorphandra mollis Benth. & $\begin{array}{l}\text { Anti-dematogenic (Mello et al., 2006); anti-toxic (Feres et al., 2006); } \\
\text { antioxidant (Petacci et al., 2010) \& anti-triptic effects (Mendes et al., } \\
2013 \text { ) }\end{array}$ & $\begin{array}{l}\text { Flavonoids (Feres et al., 2006) \& tanins (Mendes et al., } \\
\text { 2013) }\end{array}$ \\
\hline Dipteryx alata Vogel & $\begin{array}{l}\text { Anti-fungal (Nazato et al., 2010; Puebla et al., 2010; Ferraz et al., } \\
\text { 2012; Yoshida et al., 2015); non-mutagenic (Esteves-Pedro et al., } \\
\text { 2012; Yoshida et al., 2015); antioxidant, hypolipidaemic (Bento et al., } \\
\text { 2014; Fern\&es et al., 2015a); leishmanicidal effects (Ribeiro et al., } \\
\text { 2014); reduction in abdominal adiposity \& increase in HDL (de Souza } \\
\text { et al., 2018) }\end{array}$ & $\begin{array}{l}\text { Phenolic compounds \& terpenoids (Puebla et al., 2010; } \\
\text { Marques et al., 2015) }\end{array}$ \\
\hline Diptychandra aurantiaca Tul. & $\begin{array}{l}\text { No reports } \\
\text { Non-cytotoxic, non-toxic, non-mutagenic, anti-proliferative (Pinto }\end{array}$ & No reports \\
\hline Pterodon pubescens (Benth.) & $\begin{array}{l}\text { Coelho et al., 2001; Vieira et al., 2008); anti-nociceptive (Nucci et al., } \\
\text { 2012; Nucci-Martins et al., 2015); \& potential anti-inflammatory } \\
\text { effects (da Silva Santos et al., 2016; Hoscheid et al., 2017) }\end{array}$ & $\begin{array}{l}\text { Terpenes (Hoscheid et al., 2012; Nucci-Martins et al., } \\
\text { 2015) }\end{array}$ \\
\hline $\begin{array}{l}\text { Stryphnodendron adstringens } \\
\text { (Mart.) Coville }\end{array}$ & $\begin{array}{l}\text { Anti-inflammatory (Lima et al., 1998); antioxidant (Lima et al., 1998; } \\
\text { Souza et al., 2007; Santos Filho et al., 2011); antibacterial (Lima et al., } \\
\text { 1998; Souza et al., 2007; Hasenack et al., 2008; Soares et al., 2008); } \\
\text { anti-ulcerogenic (Audi et al., 1999); trypanocidal (Herzog-Soares et } \\
\text { al., 2002, 2006); anti-mutagenic (Costa et al., 2010); anti-genic } \\
\text { (Santos Filho et al., 2011) \& anti-fungal effects (de Freitas et al., } \\
\text { 2018) }\end{array}$ & $\begin{array}{l}\text { Proanthocyanidins, phenolic compounds (Palazzo de Mello } \\
\text { et al., 1996a; Palazzo de Mello et al., 1999; Costa et al., } \\
\text { 2010); prorobinetinidines (Palazzo de Mello et al., 1996b) } \\
\& \text { tannins (Santos et al., 2002) }\end{array}$ \\
\hline $\begin{array}{l}\text { Vatairea macrocarpa (Benth.) } \\
\text { Ducke }\end{array}$ & $\begin{array}{l}\text { Edematogenic (Alencar et al., 2003) \& anti-hyperglycaemic effects } \\
\text { (Oliveira et al., 2008) }\end{array}$ & No reports \\
\hline Aiouea trinervis Meisn. & $\begin{array}{l}\text { Anti-proliferative (Garcez et al., 2005a) \& trypanocidal effects (Maier, } \\
\text { 2016) }\end{array}$ & $\begin{array}{l}\text { Lignans (Garcez et al., 2005a) \& butanolide (Tsai et al., } \\
\text { 2002) }\end{array}$ \\
\hline $\begin{array}{l}\text { Ocotea minarum (Nees \& Mart.) } \\
\text { Mez }\end{array}$ & $\begin{array}{l}\text { Antioxidant, anti-microbial (Rodrigues et al., 2019) \& anti-fungal } \\
\text { effects (Rodrigues et al., 2014) }\end{array}$ & $\begin{array}{l}\text { Indole alkaloids (Vecchietti et al., 1979), phenolic } \\
\text { compounds \& terpenes (Garcez et al., 2005b) }\end{array}$ \\
\hline Strychnos pseudoquina A.St.-Hil. & $\begin{array}{l}\text { Anti-ulcerogenic (Silva et al., 2005); mutagenic (Santos et al., 2006); } \\
\text { hypoglycaemic, cicatrizative (Honório-França et al., 2008); } \\
\text { antibacterial (Bonamin et al., 2011); anti-leishmanial (Lage et al., } \\
\text { 2013); anti-HSV, anti-inflammatory (Boff et al., 2016); healing } \\
\text { (Sar\&y et al., 2018); anti-hyperglycaemic \& anti-hyperlipidaemic } \\
\text { effects (Cosenza et al., 2019) }\end{array}$ & $\begin{array}{l}\text { Alkaloids (Monache et al., 1969; \&rade-Neto et al., 2003; } \\
\text { Silva et al., 2005; Bonamin et al., 2011); flavonoids (Lage } \\
\text { et al., 2013); alkaloids, flavonoids, polyphenols \& tannins } \\
\text { (Cosenza et al., 2019) }\end{array}$ \\
\hline
\end{tabular}


Acetylcholinesterase inhibitory (Orhan et al., 2003; Rollinger et al., 2005); anti-inflammatory (Orhan et al., 2007a); antibacterial, anti-fungal (Orhan et al., 2007b); hepatoprotective (Pathak et al.,

Lycopodium clavatum $\mathrm{L}$. 2009); HeLa cell growth inhibitory (M\&al et al., 2010); human keratinocyte, skin tissue protective effects (Das et al., 2013) \& reduction in the pathogenic progression of Chagas disease (Brustolin Aleixo et al., 2017)

Cytotoxic, apoptogenic (da Silva Marcondes et al., 2014); anti-inflammatory, anti-allergic, analgesic, anti-depressant, microbicidal, antiviral, antioxidant, sedative \& anti-depressant-like, chemopreventive, angiogenic \& anti-diabetic (Carneiro et al., 2016); antibacterial, antiviral, anti-inflammatory, analgesic, anti-ulcerogenic $\&$ anti-depressant effects (Pereira et al., 2018)

Lafoensia pacari A.St.-Hil Magnolia ovata (A.St.-Hil.) $\quad$ Anti-pyretic (Kassuya et al., 2009); anti-inflammatory (Kassuya et al., Spreng. 2009; Mori et al., 2011) \& analgesic effects (Mori et al., 2011)

Alkaloids (Orhan et al., 2003, 2007a); terpenoids (Rollinger et al., 2005) \& flavonoids (Das et al., 2013) Antidiarrhoeal (Figueiredo et al., 2005); anti-mutagenic (Lira et al.,

Byrsonima basiloba A. Juss. 2008); antibacterial, anti-fungal (Michelin et al., 2008) \& antioxidant effects (Bonacorsi et al., 2013)

Mutagenic (Espanha et al., 2014); leishmanicidal (Souza et al., 2014) $\&$ antioxidant effects (Pereira et al., 2015b) Anti-microbial, anti-haemorrhagic, antidiarrhoeal, anti-inflammatory (Corrêa, 1984; Pinto \& Bertolucci, 2002; Moreira et al., 2011); anti-microbial (Michelin et al., 2008); anti-inflammatory,

Byrsonima intermedia A. Juss. anti-nociceptive (Orl\&i et al., 2011); mutagenic (Sannomiya et al., 2007), anti-ulcer, anti-microbial, antidiarrhoeal (Santos et al., 2012c); antioxidant, anti-inflammatory \& anti-peptic ulcer effects (Santos et al., 2019)

\section{Diplopterys pubipetala (A.Juss.)} W.R. Anderson \& C.C. Davis Eriotheca gracilipes (K.Schum.) A.Robyns

No reports

No reports

No reports 2012c) \& tannins (Orl\&i et al., 2011)

Flavonoids (Figueiredo et al., 2005; Lira et al., 2008)

Flavonoids (Pereira et al., 2015b)

Polyphenols (Pereira et al., 2018)

Neolignans, terpenes, alkaloids, steroids, tannins \& saponins (Kassuya et al., 2009)

Flavonoids (Pereira et al., 2015b)

Fatty acids (Mayworm \& Salatino, 1996)

Diuretic, hypotensive, anti-fungal, antioxidant, neuroprotective,

Luehea divaricata Mart. \& Zucc.

anti-inflammatory, analgesic, immunostimulatory \&

anti-cholinesterase effects (Tirloni et al., 2018)

-

Anti-microbial (Alves et al., 2000); analgesic (Vasconcelos et al., 2003); anti-inflammatory (Vasconcelos et al., 2006); DNA protective

Miconia albicans (Sw.) Triana (Serpeloni et al., 2011); antioxidant (Pieroni et al., 2011); anti-diabetic (Ortíz-Martinez et al., 2016; Lima et al., 2018); anti-microbial (Tomé et al., 2019) \& antioxidant effects (Pasta et al., 2019) Potential mutagenic (Var\&a et al., 2002; Araujo et al., 2015);

Brosimum gaudichaudii Trécul anti-microbial effects (Borges et al., 2017); stimulation of migration \& pigmentation of melanocytes (Quintão et al., 2019) \& antioxidant effects (Ferreira et al., 2019)

Antioxidant, anti-hyperlipidaemic (Ramos et al., 2007; Espindola et al., 2016); antioxidant (Vallilo et al, 2006; Coutinho et al., 2009; Pascoal et al., 2011); anti-microbial (Coutinho et al., 2009; Pavan et

Campomanesia adamantium (Cambess.) O. Berg al., 2009; Cardoso et al., 2010; Moura-Costa et al., 2012, Breda et al., 2016); anti-nociceptive (Ferreira et al., 2013); anti-proliferative (Pascoal et al., 2014; Campos et al., 2017; Ferreira et al., 2013; Fern\&es et al., 2015b; Lima e Silva et al., 2018); anti-inflammatory (Viscardi et al., 2017) \& anti-depressant effects (Souza et al., 2014) Anti-inflammatory effects (Costa et al., 2016)

Anti-fungal (Costa et al., 2000); molluscicidal (Bezerra et al., 2002); laxative (Lima et al., 2010); diarrhoeal (Lima et al., 2011; Galheigo et al., 2016); antiviral (Cecílio et al., 2012); tyrosinase inhibitory (Souza et al., 2012); gastroprotective (Prado et al., 2014); obesity preventive Eugenia dysenterica (Mart.) DC. (Donado-Pestana, 2015); anti-proliferative, acetylcholinesterase inhibitory (Gasca et al., 2017); antioxidant (Daniel Daza et al., 2017; Ferreira-Nunes et al., 2018); neuroprotective (Thomaz et al., 2018); wound healing (Silva et al., 2018); hypotensive (Fidelis-de-Oliveira et al., 2020); angiogenic \& antibacterial effects (Silva et al., 2020)

Phenolic compounds (Pavan et al., 2009; Coutinho et al., 2009; Pascoal et al., 2011, 2014; Espindola et al., 2016) No reports

Phenolic compounds, triterpenoids \& phytosterols (Tirloni et al., 2018)

Terpenes (Macari et al., 1990; Crevelin et al., 2006), phenolic compounds (Pieroni et al., 2011; Lima et al., 2018; Pasta et al., 2019); coumarins, triterpenes, tannins, flavonoids \& saponins (Tomé et al., 2019)

Coumarins (Gottlieb et al., 1972; de Morais et al., 2018; Quintão et al., 2019); condensed tannins (Monteiro et al., 2014) \& flavonoids (Araujo et al., 2015)

Terpenes (Costa et al., 2000; Duarte et al., 2009; Galheigo et al., 2016; Silva et al., 2018); saponins (Cecílio et al., 2012); tannins (Prado et al., 2014) \& flavonoids (Donado-Pestana, 2015; Silva et al., 2020) 
Anti-diabetic effects (Brunetti et al., 2006; Sales et al., 2014); recovery of the action of competitive nicotinic antagonists at the neuromuscular junction of the diaphragm of rats (Grangeiro et al., 2006); anti-inflammatory (Leite et al., 2010; Basting et al., 2014; Leite et al., 2014b; Costa et al., 2016); anti-nociceptive \& gastroprotective Eugenia punicifolia (Kunth) DC. $\quad$ effects (Basting et al., 2014); increases the exocytotic release of catecholamines from bovine adrenal chromaffin cells stimulated with ACh or K + (Pascual et al., 2011); cytostatic effects \& activation of skeletal muscle remodelling (Leite et al., 2014b); antioxidation \& inhibition of enzymes related to metabolic syndrome (Lopes et al., 2014)

$\begin{array}{ll}\text { Myrcia bella Cambess. } & \begin{array}{l}\text { Anti-mutagenic, antioxidant \& hypoglycaemic effects (Saldanha et al., } \\ \text { 2013; Vareda et al., 2014) } \\ \text { Myrcia guianensis (Aubl.) DC. }\end{array} \begin{array}{l}\text { Anti-proliferative, anti-microbial \& antioxidant effects (dos Santos et } \\ \text { al., 2018) }\end{array} \\ \text { Ouratea spectabilis (Mart.) Engl. } & \text { Antioxidant effects (Felício et al., 1995; Patel et al., 2012) } \\ \text { Myrsine umbellata Mart. } & \text { No reports } \\ \text { Roupala montana } \text { Aubl. } & \text { Schistosomicidal \& anti-genotoxic effects (Cunha et al., 2012) }\end{array}$

Roupala montana Aubl. Schistosomicidal \& anti-genotoxic effects (Cunha et al., 2012)

Alibertia edulis (Rich.) A. Rich.

Anti-microbial (Cândida da Silva et al., 2008); diuretic, hypotensive \& anti-hypertensive effects (De Santana Aquino et al., 2017)

Cordiera sessilis (Vell.) Kuntze Antioxidant \& anti-microbial effects (Aquino et al., 2013b)

Guettarda viburnoides Cham. \& Schltdl. Anti-inflammatory \& antioxidant effects (Naressi, et al. 2015)

Palicourea coriacea (Cham.) K. Schum. Anti-genotoxic (Nunes et al., 2003) \& diuretic effects (Freitas et al., 2011)

Sch

Psychotria poeppigiana Müll. Arg. Vasoactive effects (Coe \& \&erson, 1996)

$$
\text { Tocoyena brasiliensis Mart. No reports }
$$

Tocoyena formosa (Cham. \& Schltdl.) K. Schum.

Anti-fungal (Bolzani et al., 1996); antioxidant (David et al., 2007); anti-nociceptive (Cesário et al., 2018) \& anti-inflammatory effects (Cesário et al., 2019)

Anti-ulcerogenic (Basile et al., 1990; Sertié et al., 2000; de Mattos et al., 2007; Ferreira et al., 2011); anti-tumour (Itokawa et al., 1990; da Silva et al., 2008a; dos Santos et al., 2010; Felipe et al., 2014; Ferreira et al., 2016); anti-haemorrhagic \& anti-coagulant (Borges et al., 2001); anti-parasitic (Oberlies et al., 2002; Espindola et al., 2004; Antinarelli et al., 2015); genotoxic (Maistro et al., 2004); anti-inflammatory (Esteves et al., 2005; Ferreira et al., 2016; Pierri et al., 2017); anti-microbial (Schneider \& Fern\&es, 2006); anti-nociceptive (de Mattos et al., 2007); cytotoxic, anti-hyperlipidaemic (Schoenfelder et al., 2008); allelopathic (Capobiango et al., 2009); phospholipase A2 inhibitory (Ferreira et al., 2011); antioxidant (Albano et al., 2013); anti-nociceptive \& lipid-lowering effects (Brant et al., 2014) Inhibition of angiotensin converting enzyme (ACE) \& $\beta$-glucuronidase, cytotoxicity to KB cells (Arisawa et al., 1989); Allophylus edulis (A.St.-Hil. et al.) anti-hepatotoxic effects (Hoffmann-Bohm et al., 1992); negative Hieron. ionotropic effects (Matsunaga et al., 1997); antioxidant, anti-microbial, low toxic effects (Tirloni et al., 2015); antioxidant, anti-microbial \& anti-inflammatory effects (Trevizan et al., 2016) Anti-mutagenic effects (Costa et al., 2014)

Pouteria torta (Mart.) Radlk Anti-inflammatory (Vieira et al., 2003); anti-diabetogenic (Yoshikawa

Solanum lycocarpum A.St.-Hil. et al., 2007); anthelmintic (Costa et al., 2008); cytotoxic, genotoxic, anti-genotoxic (Munari et al., 2012); antioxidant (Schwarz et al., 2007) \& immunomodulating effects (Mir\&a et al., 2013) Anti-inflammatory, anti-ulcerogenic, gastric mucosal protection, analgesic, anti-convulsant, antiseptic, antibacterial, CNS depressant (Gaspi et al., 2006); mutagenic (Santos et al., 2011); antioxidant (Bonacorsi et al., 2013) \& anti-microbial effects (Pires et al., 2018)
Flavonoids (Basting et al., 2014; Sales et al., 2014), phenolic compounds \& tannins (Brunetti et al., 2006; Costa et al., 2016)

Phenolic compounds (Saldanha et al., 2013; dos Santos et al., 2018)

Polyphenolic compounds (dos Santos et al., 2018)

Phenolic compounds (Felício et al., 1995; Patel et al., 2012; Mecina et al., 2014)

No reports

Terpenes (Cunha et al, 2012); flavonods, carotenoids \& saponins (Francielli et al., 2014; Kuster \& Vale, 2016)

Flavonoids, terpenoids \& saponin (Brochini et al., 1994); tannins, alkaloids \& carotenoids (Soto-Sobenis et al., 2001; Cândida da Silva et al., 2008; Menegati et al., 2016) Terpenes, phenolic compounds \& saponins (Aquino et al., 2013b)

Iridoids (Naressi, et al. 2015)

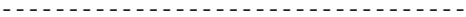
Triterpenes (Somova et al., 2003); alkaloids (Nascimento et al., 2007; do Nascimento et al., 2008); saponins, tannins \& cumarins (da Silva et al., 2008b)

Flavonoids, alkaloids, steroids, triterpenes \& coumarins (Pino-Benítez, 2006; Villasmil et al., 2006; Silva et al., 2013)

Terpenoids, saponins \& flavonoids (Hamerski et al., 2005) Iridoids, ethyl esters (Bolzani et al., 1996; Bolzani et al., 1997, Hamerski et al., 2005); polyphenolic flavonoids (Cesário et al., 2018); phenolic compounds, terpenoids \& saponins (Cesário et al., 2019)

Flavonoids (Bueno et al., 2016; Vieira Júnior et al., 2017) $\&$ terpenes (Basile et al., 1990; Oberlies et al., 2002; Espindola et al., 2004; Santos et al., 2007)

Triterpenoids, alkaloids \& anthocyanidins (B\&oni et al., 1976); cyanolipid, triacylglycerol (Aichholz et al, 1997); phenolic compounds (Arisawa et al., 1989; Hoffmann-Bohm et al., 1992); L-quebrachitol (Díaz et al., 2008) \& terpenes (Trevizan et al., 2016)

Flavonoids (Costa et al., 2014)

Phenols, tannins, flavonoids, steroids, terpenes, saponins, alkaloids (Araújo et al., 2010; Munari et al., 2012; Torralbo et al., 2012) \& pectin (Torralbo et al., 2012)

No reports 


\begin{tabular}{lll} 
Qualea multiflora Mart. & $\begin{array}{l}\text { Molluscicidal (de Souza et al., 1984); cytotoxic (Nasser et al., 2008) \& } \\
\text { mutagenic effects (Santos et al., 2011) }\end{array}$ & $\begin{array}{l}\text { Terpenes, steroids (Santos et al., 2011) \& ellagic acid } \\
\text { derivatives (Nasser et al., 2008; Carnevale Neto et al., }\end{array}$ \\
& $\begin{array}{l}\text { Antioxidant (Bonacorsi et al., 2013); } \text { in vitro mutagenic (Santos et al., } \\
\text { Qualea parviflora } \text { Mart. }\end{array}$ & $\begin{array}{l}\text { 2011); gastroprotective, antidiarrhoeal, anti-haemorrhagic \& } \\
\text { mutagenic effects (Mazzolin et al., 2010) }\end{array}$ \\
Vochysia tucanorum Mart. & Gastroprotective effects (Gomes et al., 2009) & $\begin{array}{l}\text { Polyphenols, flavonoids \& condensed tannins (Franco et } \\
\text { al., 2019) }\end{array}$ \\
\hline
\end{tabular}

\subsection{Brazilian Government-Public Access to Herbal Medicines}

In Brazil, the Unified Health System (SUS) is in place and consists of a public health system that covers everything from primary care to organ transplantation, guaranteeing full, universal and free access by the entire country's population. Among the existing projects of the SUS is the RENISUS (National List of Medicinal Plants of Interest to the SUS), which contains medicinal plants that have the potential to generate products of interest to the SUS. Among the listed species are 71 plants popularly used and scientifically confirmed. The purpose of the list is to guide studies and research that can support elaboration of the list of herbal medicines available for safe and effective use by the population to treat a certain disease (De Aquino et al., 2019).

Considering the relevance of the plant species found in the RENISUS list, we researched which were present in the studied area (Settlement 17 de Abril) and related them to their popular uses and associated scientific studies. Of the 89 species found in the April 17 settlement, only three are listed in the RENISUS: Casearia sylvestris, Copaifera spp * (Copaifera langsdorffii) and Stryphnodendron adstringens.

Casearia sylvestris, popularly known as "guaçatonga", is used in folk medicine for purification and to treat diarrhoea, fever, rheumatism, skin disorders and snake bites (Table 1). Scientific studies report anti-ulcerogenic, anti-inflammatory, anti-tumour, anti-hyperlipidaemic, anti-coagulant, trypanocidal, leishmanicidal, anti-microbial, genotoxic, allelopathic, anti-hyperalgesic, antioxidant, phospholipase A2 inhibitory and anti-parasitic effects of leaves (Table 2). The bark demonstrated anti-tumour activity (Table 2). Phytochemical studies report the presence of flavonoids in the leaves and aerial parts and terpenes in the leaves of this species (Table 2).

Copaifera langsdorffii is popularly known as "copaiba", and resin-extracted oil from its trunk is used by the population to treat inflammation, sore throat, urinary and pulmonary infections, and accelerate wound and ulcer healing (Table 1). Studies conducted on the leaves of this species report potential gastroprotective, anti-inflammatory and antifungal effects and significant genotoxicity (Table 2). Phytochemical studies reported the presence of galloquinquinic acids in the leaves. Oil extracted from the trunk, which is popularly used in folk medicine, has been shown in biological studies to induce cell cycle arrest and apoptosis, with a protective effect against colon carcinogenesis, cytotoxicity, embryotoxicity and bacteria (Table 2). The seeds present immunomodulatory activity and the presence of xyloglucans and galloquinquinic acids (Table 2). Another part of this species is the fruits, which present antioxidant activity (Table 2).

The barbed bark from Stryphnodendron adstringens, "barbatimão", is widely used in two different ways: externally for the treatment of uterine conditions, vaginal conditions, urinary tract infections, skin lesions, ulcers, inflammation, infections and skin infections; and internally for diarrhoea, throat inflammation, bleeding, scurvy, pulmonary complications, and respiratory infections. Additionally, internal use can be used to treat diarrhoea, sore throat, bleeding, scurvy, pulmonary complications, and respiratory infections (Table 1). Scientific studies have validated the popular indications, highlighting some biological activities in the stem bark of this species. Studies report trypanocidal, anti-fungal, antibacterial, antioxidant, anti-ulcerogenic, antigenic, anti-inflammatory and anti-mutagenic effects, as well as the presence of proanthocyanidins, flavonoids, prorobinetinidines and tannins (Table 2).

The presence of these species demonstrates the importance of further scientific studies to prove their ethnopharmacological action against diseases, as well as ensuring secure access to and the rational use of medicinal and herbal plants, the development of technologies and innovations, the strengthening of chains and productive arrangements, the sustainable use of Brazilian biodiversity and the development of the Health Productive Complex.

\section{Conclusion}

This study provides 89 medicinal plants belonging to 39 families documented from the study area (reserve area of the settlement "17 April", MS, Brazil) for the first time. The most widespread traditional use, involves the 
treatment of gastro-intestinal system diseases (41 spp) and the most utilized parts were leaves (41\%), justifying the conservation of biodiversity. Thus, future studies should be focused to establish the links between the traditional uses, active compounds and reported pharmacological activities. Additionally, three species are listed in the National Program for Medicinal and Phototherapeutic Plants (Casearia sylvestris, Copaifera langsdorffii and Stryphnodendron adstringens).

\section{References}

Amaral, A., Munhoz, C., Walter, B., Aguirre-Gutiérrez, J., \& Raes, N. (2017). Richness pattern and phytogeography of the Cerrado herb-shrub flora and implications for conservation. Journal of Vegetation Science, 28, 848-858. https://doi.org/10.1111/jvs.12541

De Aquino, C. M. F., de Sousa, I. M. C., Assunção, M. C. T., Neto, M. R. C., \& Bezerra, A. F. B. (2019). Cost Comparison of Integrative and Complementary Practices Specialized Unit in SUS. Advances in Integrative Medicine, 6, S133. https://doi.org/10.1016/j.aimed.2019.03.388

Di Stasi, L. C., Oliveira, G. P., Carvalhaes, M. A., Queiroz, M. Jr., Tien, O. S., Kakinami, S. H., \& Reis, M. S. (2002). Medicinal plants popularly used in the Brazilian Tropical Atlantic Forest. Fitoterapia, 73, 69-91. https://doi.org/10.1016/S0367-326X(01)00362-8

Durigan, G., \& Ratter, J. (2015). The need for a consistent fire policy for Cerrado conservation. Journal of Applied Ecology, 53. https://doi.org/10.1111/1365-2664.12559

Guerra, A., Roque, F. O., Garcia, L. C., Ochao-Quintero, J. M. O., de Oliveira, T. S., Guariento, R. D., \& Rosa, I. M. D. (2020). Drivers and projections of vegetation loss in the Pantanal and surrounding ecosystems. Land Use Policy, 91, 104388. https://doi.org/10.1016/j.landusepol.2019.104388

Kirk, M. D., Angulo, F. J., Havelaar, A. H., \& Black, R. E. (2017). Diarrhoeal disease in children due to contaminated food. Bulletin of World Health Organazation, 95, 233-234.

Sano, E. E., Rodrigues, A. A., Martins, E. S., Bettiol, G. M., Bustamante, M. M. C., Bezerra, A. S., ... Bolfe, E. L. (2019). Cerrado ecoregions: A spatial framework to assess and prioritize Brazilian savanna environmental diversity for conservation. Journal of Environmental Management, 232, 818-828. https://doi.org/10.1016/ j.jenvman.2018.11.108

Strassburg, B. B. N., Latawiec, A. E., Barioni, L. G., Nobre, C. A., da Silva, V. P., Valentim, J. F., ... Assad, E. D. (2014). When enough should be enough: Improving the use of current agricultural lands could meet production demands and spare natural habitats in Brazil. Global Environmental Change, 28, 84-97. https://doi.org/10.1016/j.gloenvcha.2014.06.001

\section{Abbreviations}

CSD, Cardiovascular System Diseases; DDMS, Protocol number specimen; DSD, Gastro-intestinal System Diseases; ENM, Endocrine System Diseases; ID, others Inflammatory Diseases; IPD, Infectious Diseases; GUS, Obstetrics, Gynecology and Urinary-tract Diseases; MCT, Musculoskeletal and Joint Diseases; MS, Mato Grosso do Sul; NEP, Malignant Diseases; NSD, Central Nervous System Diseases; OD, Others Diseases; RENISUS, National list of medicinal plants of interest to the SUS; RSD, Respiratory System Diseases; SUS, Unified health system; SST, Skin, Eye, Ear, Nose and Oropharynx Diseases; WHO, World Health Organization.

\section{Supplementary Files}

References listed in Tables 1 and 2 can be retrieved from http://admin.ccsenet.org/press/index.php/galley/down load/article/43938/id/46206

\section{Copyrights}

Copyright for this article is retained by the author(s), with first publication rights granted to the journal.

This is an open-access article distributed under the terms and conditions of the Creative Commons Attribution license (http://creativecommons.org/licenses/by/4.0/). 